\title{
Perfil cognitivo de los pacientes con traumatismo craneoencefálico grave: un estudio a los dos años de evolución
}

\section{Cognitive profiles of patients with severe traumatic brain injury: study at two years follow-up}

\author{
Enrique Moraleda-Barreno ${ }^{1,2 *}$, Modesto Jesús Romero-López ${ }^{1,2}$, María José Cayetano-Menéndez ${ }^{1,2}$ \\ 1 Servicio de Evaluación y Rehabilitación Neuropsicológica. Huelva, España. \\ 2 Universidad de Huelva. Huelva, España.
}

\section{Resumen}

Las alteraciones cognitivas de los sujetos con traumatismos craneoencefálicos (TCE) han sido estudiadas ampliamente en fase aguda y post-aguda. El objetivo del presente trabajo es obtener el perfil cognitivo de los pacientes con TCE graves a los dos años de evolución. La muestra de estudio estuvo compuesta por 42 sujetos adultos con TCE cerrado grave, a los que se les realizó una evaluación neuropsicológica que abarcó un amplio número de funciones cognitivas. Se comparó la ejecución de los sujetos con las puntuaciones estándar para la población normal. Los resultados mostraron una disminución generalizada de todas las funciones cognitivas evaluadas: inteligencia general, atención, visuopercepción y razonamiento visual, memoria, aprendizaje, funciones verbales, destrezas académicas, visuoconstrucción y funciones ejecutivas. La atención compleja es, con diferencia, la función más afectada. Esto tiene repercusiones importantes en la rehabilitación cognitiva de estos pacientes.

Palabras clave: alteraciones cognitivas, atención compleja, evaluación neuropsicológica, neuropsicología, traumatismos craneoencefálicos

\begin{abstract}
Cognitive impairments of the patients with traumatic brain injury (TBI) have been studied in the first year of evolution extensively. The objective of this work is to study the cognitive deficits of the TBI after two years at follow up. We analyzed 42 adult patients with severe closed TBI that underwent a neuropsychological assessment of a wide range of cognitive functions. We compared the performance of the patients with the scores standard for the normal population. The results show a decrease in performance of all cognitive functions: general intelligence, attention, visual visuoperception and reasoning, memory, learning, verbal functions, academic skills, and executive functions. The lowest scores are given on the function of complex attention. This has important implications for cognitive rehabilitation of patients with closed TBI.
\end{abstract}

Keywords: cognitive deficits, complex attention, neuropsychological assessment, neuropsychology, traumatic brain injury

\footnotetext{
*Correspondencia: enrique.moraleda@dpsi.uhu.es. Departamento de Psicología Clínica, Experimental y Social. Facultad de Ciencias de la Educación. Campus El Carmen, Avd. Tres de Marzo, s/n 21071 Huelva, España Recibido: 14-03-2011. Revisión desde: 26-03-11. Aceptado: 06-07-11
}

DOI: 10.5839/rcnp.2011.0601.07 


\section{Introducción}

Los avances producidos en diversas especialidades de la medicina han logrado un incremento notable de la supervivencia en pacientes con traumatismos craneoencefálicos (TCE) en las últimas décadas, lo cual conlleva un aumento de la prevalencia de graves secuelas de tipo neuropsicológico que son las que más dificultan la reintegración familiar, social y laboral (Junqué, Bruna y Mataró, 1998; Lezak, Howieson, Loving, Hannay \& Fisher, 2004). Los TCE son uno de los principales problemas sociales y sanitarios, con una incidencia de entre 200 y 300 casos cada 10.000 habitantes (Muñoz-Céspedes, PaulLapedriza, Pelegrín-Valero \& Tirapu-Ustarroz, 2001; Tagliaferri, Compagnone, Korsic, Servadei \& Kraus, 2006).

Un factor pronóstico de las secuelas cognitivas es la gravedad del traumatismo (Bode, Heinerman \& Semik, 2000), que se pronostica en función de tres principales parámetros: la puntuación en la Glasgow Coma Scale (GCS), la duración del coma y el periodo de amnesia postraumática (Muñoz- Céspedes et al., 2001). Se considera que un TCE es grave cuando la puntuación en la GCS es de 3-8 puntos, el periodo de amnesia postraumática es igual o superior a 24 horas y el tiempo de coma superior a 6 horas.

Otro factor necesario de tener en cuenta es la naturaleza de la lesión (De la Cueva et al., 2009), siendo las lesiones de peor pronóstico las de mayor extensión, ya que afectan a mayor masa cerebral. Entre estas se encuentran las difusas, intracerebrales, bihemisféricas, frontotemporales, subcorticales y troncoencefálicas (Marshall et al., 1988; Englander, Cifu, Wright \& Black, 2003; Wardlaw, Easton \& Statham, 2002).

Las secuelas cognitivas se deben principalmente al daño cerebral provocado por mecanismos primarios (contusiones, hematomas, edemas y lesión axonal difusa) e incluyen déficit tanto focales como difusos. Entre los déficit focales se encuentran las afasias, apraxias, agnosias, alexias, acalculias y amnesias (Junqué et al., 1998). Los déficit difusos incluyen la disminución del nivel de conciencia, alteraciones en memoria, atención, funciones ejecutivas, el razonamiento, percepción y lenguaje (Nickels, Schneider, Donbovy \& Wong, 1994; Perea, Ladera \& Morales, 1999; Prigatano, 1999). Así, como consecuencia de un TCE, todas las funciones neuropsicológicas pueden resultar afectadas (Caracuel \& Cuberos, 2009).

Después del primer año del TCE son frecuentes los déficit en el funcionamiento cognitivo (Junqué et al., 1998), especialmente en memoria, concentración, velocidad de procesamiento y resistencia a la fatiga (Tabaddor, Mattis \& Zazula, 1984; Van Zomeren \& Van der Burg, 1985; Verger et al., 2001). Las funciones con mejor evolución, una vez pasados 5 años, son la memoria verbal, la capacidad constructiva y la velocidad de procesamiento, aunque es preciso tener en cuenta que las secuelas a largo plazo no son uniformes entre sujetos ni entre tipos de lesiones (Millis et al., 2001).

En el caso de los TCE cerrados, se suelen producir dos tipos de efectos sobre la conducta: un deterioro de las funciones específicas controladas por la zona lesionada de la corteza cerebral (generalmente los lóbulos frontal y temporal) y deterioros más generalizados debidos a la afectación causada por el movimiento de los hemisferios (contusiones, laceraciones dispersas y desgarros), como la pérdida de funciones cognitivas complejas, concretamente la reducción de la velocidad mental, la concentración y la eficiencia cognitiva global (Kolb \& Wishaw, 2006).

Respecto de la memoria, si bien numerosos estudios han encontrado trastornos en la memoria declarativa, resultando preservado el aprendizaje procedimental (Timmerman \& Brower, 1999; Ewert, Levin, Watson \& Kalisky, 1987; Shum, Sweper y Murray, 1993; Wilson, 1992), no ha sido así en otras investigaciones, lo que sugiere que, aunque la memoria procedimental resulte más resistente al daño, también puede verse afectada en muchos casos (Verger et al., 2001).

Los problemas atencionales son muy comunes y afectan a la atención sostenida, la distractibilidad, la interferencia, los tiempos de reacción, la desinhibición de respuestas inapropiadas, la incapacidad para continuar las actividades iniciadas, la limitación para simultanear varias acciones o la alternancia de la atención (Ríos-Lago, MuñozCéspedes \& Paul-Lapedriza, 2007). También es frecuente la fatiga mental provocada por el esfuerzo necesario para superar los trastornos atencionales (Ziino \& Ponsford, 2006).

Las funciones ejecutivas se ven afectadas en el manejo de situaciones cambiantes, la adquisición de nuevos aprendizajes, la inconsistencia en el uso de estrategias en situaciones similares y la capacidad para integrar los conocimientos y aplicarlos a situaciones concretas (Muñoz-Céspedes et al., 2001; Von Cramon \& Von Cramon, 1992; Hancks, Rapport, Millis \& Deshpande, 1999). Estos déficit afectan a la planificación, la resolución de problemas, el pensamiento abstracto, la flexibilidad mental y el control del pensamiento y la conducta (Ariza, Pueyo \& Sierra, 2004).

También se producen, aunque con menos frecuencia, alteraciones de la percepción visual, la integración de la información sensorial y las funciones visuoconstructivas (Caracuel \& Cuberos, 2009; Cullum \& Bliger, 1986).

Tras un TCE, el lenguaje suele ser tangencial, poco organizado y con dificultades pragmáticas. Las alteraciones más frecuentes del lenguaje y el habla en este tipo de lesiones son la anomia (Levin \& Heisenberg, 1979) y la falta de fluidez verbal (Lezak et al., 2004), siendo los diagnósticos principales la disartria y la afasia subclínica con disartria o afasia clásica (Sarno, Buenafuora \& Levita, 1986). En las lesiones focales que afectan a áreas concretas del lenguaje pueden presentarse trastornos en aspectos como la comprensión, expresión, repetición, denominación, lectoescritura y articulación (Junqué et al., 1998).

En cuanto al cociente de inteligencia general, los pacientes leves y con buena recuperación suelen situarse en niveles normales o límite, mientras que los pacientes graves suelen mostrar alteraciones importantes (Junqué et al., 1998), resultando más afectados los subtest manipulativos que los verbales (Levin \& Heisenber, 1979).

La evaluación neuropsicológica de los TCE debe incluir la valoración de la atención, memoria, aprendizaje, lenguaje, funciones ejecutivas, velocidad de procesamiento, razonamiento, habilidades comunicativas y funciones perceptivas y constructivas (Lezak et al., 2004). Aunque existen baterías generales de evaluación, es preferible la utilización de baterías 
compuestas por pruebas que evalúen diferentes funciones específicas en profundidad (Junqué et al., 1998).

Se puede concluir que el TCE causa daño cerebral focal o difuso que afecta a una amplia gama de funciones cognitivas. Las secuelas cognitivas originadas se han estudiado ampliamente en la fase aguda y post-aguda del TCE, y no tanto en la fase crónica. En este trabajo se analizan las distintas funciones cognitivas afectadas en personas con TCE cerrado a los dos años de evolución. El TCE cerrado causa daño cerebral difuso, por lo que se deben esperar secuelas cognitivas generalizadas debido a la naturaleza de la lesión.

\section{Método}

\section{Participantes}

Participaron en este estudio un total de 42 pacientes (36 varones y 6 mujeres) que habían sufrido un TCE cerrado grave por accidente de tráfico. Los criterios de inclusión de la muestra de estudio fueron: una puntuación inferior o igual a 8 en la escala de coma de Glasgow al ingreso hospitalario tras el accidente, una pérdida de conciencia superior a 6 horas, una duración del periodo de amnesia postraumática superior a 24 horas y un periodo medio de ingreso en cuidados intensivos de 19 días. Un 26,6\% presentaban lesión frontal, 42,9\%, además de en el lóbulo frontal, presentaba lesiones en el parietal o el temporal, $4,8 \%$ solo tenía afectación temporal y otro $4,8 \%$ parietal; $16,7 \%$ presentaba lesión axonal difusa. El $31 \%$ de los sujetos mostró lesión en el hemisferio derecho, 40,5\% en el izquierdo y $28,6 \%$ sufría lesión bilateral. La edad media de los sujetos es de $33.3 \pm 14.3$ años. Se excluyeron los que presentaban síndrome afásico debido a la imposibilidad para la realización de algunas pruebas. Todos los sujetos dieron su consentimiento informado y participaron voluntariamente en este estudio.

\section{Instrumentos}

La valoración neuropsicológica incluyó las siguientes pruebas: la Escala de inteligencia de Weschler para adultos (WAIS) (Wechsler, 1999), y el Test de inteligencia verbal Toni-2 (Brown, Sherbenou \& Johnsen, 1995) como medida de la inteligencia general; el Trail making test (TMT-A y B) (Reitan, 1958) el Test de dígitos y símbolos (SDMT) (Smith, 2002) y la aritmética, dígitos y clave de números de la WAIS para la atención; los subtest figuras incompletas e historietas de la WAIS para la visuopercepción y el razonamiento visual; la Curva de memoria de Luria (Ardila, Roselli \& Puente, 1994) para la memoria verbal; el Test de Corsi (CBTT) (Milner, 1986) y el Test de retención visual de Benton (TRVB) forma C, administración A (Benton, 1986; Ladera, Perea y Morales, 2002) para la memoria y el aprendizaje; los subtest información, comprensión y semejanzas de la WAIS y el test de fluidez verbal (FV) (Benton, 1977) para las funciones verbales y destrezas académicas; el subtest de cubos de la WAIS para la visuoconstrucción; el Test de clasificación de tarjetas de Wisconsin (WCST) (Heaton, Chelune, Talley \& Curtiss, 2001) y los subtest de historietas y rompecabezas como medida de las funciones ejecutivas.
Se realizó una evaluación neuropsicológica a los dos años tras el accidente (20-28 meses). Se estudiaron los rendimientos en inteligencia general, atención, visuopercepción y razonamiento visual, memoria y aprendizaje, funciones verbales y destrezas académicas, visuoconstrucción y funciones ejecutivas. Los sujetos fueron evaluados en un número de sesiones que osciló entre cuatro y siete. Las sesiones constaban de estudio de la historia clínica siguiendo los protocolos del servicio donde se realizaron las evaluaciones neuropsicológicas, entrevistas con los sujetos y familiares y realización de las diferentes pruebas. Se compararon los resultados de los sujetos con los valores normativos ofrecidos por las diferentes pruebas. Se estudió la relación de las secuelas cognitivas con la localización y lateralización del daño cerebral.

\section{Resultados}

No se encontraron diferencias estadísticamente significativas al comparar los diferentes grupos de sujetos según la localización de la lesión, ya fuese esta frontal, temporal, parietal, lesión axonal difusa o abarcara al mismo tiempo el lóbulo frontal y otro. Tampoco, según la lateralización de la lesión. Los resultados para la localización y lateralización del daño cerebral se muestran en la tabla 1.

El resultado más destacable del estudio de las diferentes funciones es que las puntuaciones medias de los sujetos en todas las pruebas son inferiores a las de la población normal reflejada en los baremos. Las pruebas en las que se observó una peor ejecución son TMT-A, TMT-B y Test de Corsi, mientras que las menos afectadas fueron WCST, CML Y semejanzas de la WAIS. En la tabla 2 se presentan las puntuaciones de los sujetos en las diferentes test. A continuación se describen los resultados según la función cognitiva comprometida en cada grupo de pruebas.

Inteligencia general: En la WAIS total las puntuaciones fueron 1.3 desviaciones típicas inferiores a la media, en la verbal de 1.1 y en la manipulativa de 1.4. En el Toni-2 las puntuaciones fueron de 0.8 DT por debajo de la media. No hubo diferencias significativas entre los $\mathrm{Cl}$ verbales y manipulativos.

Atención: En el TMT-A y B destaca un elevado tiempo de ejecución, 9.7 DT por encima de la media. En SDMT los sujetos obtienen un número de respuestas correctas que se encuentra $2 D T$ por debajo de lo esperado (clasificada como muy baja). En las subpruebas de la WAIS las puntuaciones también son bajas. Aritmética se encuentra 0.9 DT por debajo de la media, dígitos 0.8 y clave de números 1.5.

Visuopercepción y razonamiento visual: Las puntuaciones se encuentran claramente por debajo de la media (figuras incompletas 1.4 DT; e historietas 1.4 DT).

Memoria y aprendizaje: En la Curva de memoria de Luria las puntuaciones están 0.5 DT por debajo de la media. En el Test de Corsi la puntuación es 4.3 DT inferior a la media y en el Test de retención visual de Benton de 1.5.

Funciones verbales y destrezas académicas: En los subtest información y semejanzas las puntuaciones no llegan a ser una $D T$ por debajo de la media ( 0.7 y 0.5 respectivamente), mientras que comprensión es inferior en $1.2 \mathrm{DT}$. El test de fluidez verbal está $0.8 D T$ por debajo de la media.

\section{Procedimiento}


Visuoconstrucción: Las puntuaciones en el subtest cubos se encuentran 0.9 DT por debajo de la media.

\begin{tabular}{|c|c|c|c|c|c|c|c|c|}
\hline & \multicolumn{4}{|c|}{ Localización lobar* } & \multicolumn{4}{|c|}{ Lateralidad** } \\
\hline & $\mathrm{F}$ & gl 1 & $\mathrm{gl} 2$ & Sig & $\mathrm{F}$ & gl 1 & gl 2 & Sig \\
\hline WAIS verbal & 1.264 & 5 & 36 & .305 & 1.428 & 2 & 39 & .254 \\
\hline WAIS manipulativo & 1.072 & 5 & 36 & .398 & .810 & 2 & 39 & .455 \\
\hline WAIS total & .990 & 5 & 36 & .443 & 1.765 & 2 & 39 & .189 \\
\hline Toni-2 & 2.138 & 5 & 36 & .083 & 1.405 & 2 & 39 & .257 \\
\hline WCST Pep & .595 & 5 & 36 & .704 & 649 & 2 & 39 & .530 \\
\hline TMT-A tiempo & .379 & 5 & 36 & .859 & 1.376 & 2 & 39 & .268 \\
\hline TMT-B tiempo & .486 & 5 & 36 & .783 & .830 & 2 & 39 & .446 \\
\hline SDMT correctas & .516 & 5 & 36 & .678 & .859 & 2 & 39 & .443 \\
\hline Test de Corsi & .923 & 5 & 36 & .484 & 1.200 & 2 & 39 & .317 \\
\hline TRVB correctas & .845 & 5 & 36 & .534 & .642 & 2 & 39 & .536 \\
\hline Fluidez verbal & .613 & 5 & 36 & .692 & .615 & 2 & 39 & .550 \\
\hline CML & .041 & 5 & 36 & .997 & 2.174 & 2 & 39 & .135 \\
\hline
\end{tabular}

Tabla 1. Comparación de los resultados de las pruebas en función de la localización lobar y la lateralidad de la lesión.

* La columna localización muestra los resultados de la prueba ANOVA de un factor para la comparación entre los resultados de los sujetos con diferentes localizaciones lobulares de la lesión (frontal, frontal y otra, temporal, parietal y lesión axonal difusa).

** La columna lateralidad muestra los resultados de la prueba ANOVA de un factor para la comparación entre los resultados de los sujetos con diferente lateralización de la lesión (derecha, izquierda y bilateral).

\begin{tabular}{|c|c|c|c|c|}
\hline Pruebas & Media Sujetos & $D T$. Sujetos & Media Estándar & DT. Estándar \\
\hline WAIS verbal & 87.3 & 17.1 & 103.4 & 14.5 \\
\hline WAIS manipulativo & 88.1 & 15.4 & 108.3 & 14.4 \\
\hline WAIS total & 87.7 & 15.6 & 105.8 & 14.3 \\
\hline Vocabulario & 8.7 & 2.5 & 10.8 & 2.8 \\
\hline Semejanzas & 9.8 & 3.8 & 11.3 & 2.7 \\
\hline Aritmética & 7.7 & 2.8 & 10.1 & 2.7 \\
\hline Dígitos & 7.9 & 2.4 & 10.4 & 3.1 \\
\hline Información & 8.4 & 3.1 & 10.5 & 2.8 \\
\hline Comprensión & 7.6 & 3.3 & 11 & 2.9 \\
\hline Figuras incompletas & 7.5 & 3.1 & 11.1 & 2.6 \\
\hline Clave de números & 7.4 & 3.5 & 11.8 & 3 \\
\hline Cubos & 8.6 & 3.5 & 11.4 & 2.9 \\
\hline Historietas & 7.2 & 2.6 & 11.1 & 2.8 \\
\hline Rompecabezas & 7.7 & 2.8 & 11.3 & 3.1 \\
\hline Toni-2 & 88.1 & 29.6 & 100 & 15 \\
\hline WCST Pep & 94 & 41.5 & 100 & 15 \\
\hline TMT-A tiempo & 130.7 & 143.5 & 27.5 & 8.3 \\
\hline TMT-B tiempo & 231.9 & 158.8 & 62.1 & 17.5 \\
\hline SDMT correctas & 24.4 & 13.2 & 49.2 & 8.79 \\
\hline Test de Corsi & 4.2 & 0.7 & 10.9 & 1.6 \\
\hline TRVB correctas & 5 & 2.7 & 7.2 & 1.4 \\
\hline Fluidez verbal & 15.4 & 8.9 & 24.5 & 11.9 \\
\hline CML & 5.9 & 2.1 & 6.7 & 1.5 \\
\hline
\end{tabular}

Tabla 2. Puntuaciones medias y desviaciones típicas de los sujetos evaluados y la población sin daño cerebral. En las dos primeras columnas se muestran las puntuaciones medias y desviaciones típicas de los sujetos evaluados, en las dos últimas aparecen las medias y desviaciones típicas de la media de la población en las diferentes pruebas. 


\begin{tabular}{|c|c|c|c|c|c|}
\hline Pruebas & $<-2 \mathrm{DT}$ & $-2<-1 \mathrm{DT}$ & $-1<0$ DT & $0<1 \mathrm{DT}$ & $>1 \mathrm{DT}$ \\
\hline CI WAIS verbal & 17 & 17 & 44 & 22 & 0 \\
\hline CI WAIS manipulativo & 12 & 24 & 47 & 18 & 0 \\
\hline CI WAIS total & 18 & 18 & 41 & 24 & 0 \\
\hline Vocabulario & 0 & 33 & 44 & 18 & 5 \\
\hline Semejanzas & 14 & 5 & 31 & 36 & 14 \\
\hline Aritmética & 10 & 33 & 43 & 14 & 0 \\
\hline Dígitos & 9 & 36 & 27 & 28 & 0 \\
\hline Información & 16 & 21 & 37 & 21 & 5 \\
\hline Comprensión & 10 & 45 & 25 & 10 & 10 \\
\hline Figuras incompletas & 26 & 5 & 58 & 11 & 0 \\
\hline Clave de números & 26 & 16 & 42 & 16 & 0 \\
\hline Cubos & 14 & 23 & 27 & 32 & 5 \\
\hline Historietas & 11 & 47 & 26 & 16 & 0 \\
\hline Rompecabezas & 6 & 22 & 61 & 11 & 0 \\
\hline Toni-2 & 39 & 26 & 18 & 9 & 8 \\
\hline WCST P.E.P. & 34 & 13 & 12 & 18 & 23 \\
\hline TMT-A tiempo & 90 & 10 & 0 & 0 & 0 \\
\hline TMT-B tiempo & 84 & 16 & 0 & 0 & 0 \\
\hline SDMT correctas & 40 & 60 & 0 & 0 & 0 \\
\hline Test de Corsi & 100 & 0 & 0 & 0 & 0 \\
\hline TRVB correctas & 27 & 44 & 11 & 9 & 9 \\
\hline Fluidez verbal & 0 & 38 & 46 & 16 & 0 \\
\hline CML & 19 & 38 & 25 & 13 & 5 \\
\hline
\end{tabular}

Tabla 3. Distribución de las puntuaciones de los sujetos en los distintos test en porcentajes. Puede observarse que, en la gran mayoría de los sujetos, el rendimiento se encuentra por debajo de la media.

Funciones ejecutivas: La puntuación de porcentaje de errores perseverativos del Test de clasificación de tarjetas de Wisconsin (WCST-pep) el resultado es un 0.4 DT inferior a la media. El subtest de historietas, puntúa un 1.4 DT por debajo de la media y el de rompecabezas un 1.2.

La distribución de las puntuaciones de los sujetos en desviaciones típicas se muestra en la tabla 3. Las pruebas en las que un mayor número de sujetos presentan puntuaciones muy bajas (inferiores a 1 DT) son Toni-2, WCST Pep, TMT-A, TMT-B, SDMT, CML, TRVB, comprensión, historietas y, sobre todo, el Test de Corsi, en el que todos los sujetos fracasan. Por el contrario, una gran mayoría obtiene puntuaciones dentro de la media en los $\mathrm{Cl}$ verbal, manipulativo y total de la WAIS.

\section{Discusión}

Los resultados muestran que estos pacientes sufren un deterioro generalizado de las capacidades cognitivas propio de las lesiones generales difusas. Este tipo de daño puede explicar que, al comparar los diferentes grupos de sujetos según la localización y lateralización del daño cerebral, no se encuentran diferencias estadísticamente significativas en las funciones cognitivas.

Como se observa, en casi todas las pruebas se obtienen resultados que se sitúan en torno a una desviación típica por debajo del baremo correspondiente a la población normal. Se podría concluir que se ven disminuidas todas las funciones cognitivas, aunque la significativamente más afectada es la atención. Este resultado es predominante en las pruebas que requieren atención compleja, las cuales se basan en tareas que requieren concentración, atención sostenida y focalizada, alternancia del foco atencional, coordinación psicomotora y velocidad de reacción (Lezak et al., 2004), por lo que son muy sensibles al daño cerebral y su disfunción es típica de las lesiones producidas por deceleración rápida, tal como ocurre en los TCE causados por accidentes de tráfico. Así, hemos encontrado que, tanto en el TMT como en el SDMT, un gran número de sujetos obtienen puntuaciones dos o más desviaciones típicas por debajo de la media de su grupo normativo, al igual que en otras pruebas como el Test de Corsi, con un gran componente atencional aparte del visoespacial.

También se observa un elevado tiempo de ejecución en todas las pruebas, lo cual se interpreta como un 
enlentecimiento en el procesamiento de la información (Tromp \& Mulder, 1991). Los datos muestran que los pacientes rinden peor en las pruebas que requieren un determinado tiempo de ejecución, siendo pésimo el rendimiento en las tareas más complejas que precisan más recursos atencionales. En general, estos déficit disminuyen el rendimiento en general y afectan a otras funciones cognitivas implicadas, como la formación de conceptos o el razonamiento complejo (Ariza et al., 2004).

La memoria de trabajo está estrechamente relacionada con esas funciones atencionales y también se encuentra deteriorada, tanto en su modalidad verbal como en la espacial (Test de Corsi) y la visuoperceptiva (TRVB). También se aprecian dificultades en la evocación de palabras que sugieren un déficit de memoria semántica. Estos datos son consistentes con los de otros autores que encuentran déficit de memoria, tanto verbal como no verbal, más allá del año de producirse la lesión (Ladera et al., 2002; Valik, 2005).

Los pacientes muestran dificultades al realizar tareas que requieren el análisis y procesamiento de estímulos visuales, así como la manipulación de objetos en tareas de construcción que necesitan el manejo de datos visuoperceptivos y visuoespaciales.

No se encuentran déficit verbales significativos, aunque tampoco se esperaban, ya que se excluyeron de la muestra los sujetos que sufrían afasia. Aun así, la ejecución en este tipo de tareas verbales se encuentra por debajo de ejecución media de la población normal. Las puntuaciones en las destrezas académicas se encuentran dentro de lo esperado, aunque con un sesgo hacia puntuaciones bajas. Esto puede deberse a la influencia de los déficit generalizados que muestra la mayor parte de los sujetos.

La alteración de las funciones ejecutivas se muestra en las pruebas que requieren autocorrecciones y flexibilidad en la respuesta, como el WCST, en el cual se observa un alto número de perseveraciones. Los pacientes muestran dificultades en las tareas que requieren una organización y acción propositiva, como ocurre con los subtest de historietas y rompecabezas de la WAIS. Estos déficits típicos de las lesiones frontales también están presentes en las lesiones difusas y se ven incrementados por los trastornos atencionales.

La distribución de los sujetos en las pruebas de inteligencia muestra que solo $24 \%$ de los pacientes obtienen puntuaciones por encima de la media. Esto sugiere una disminución general del cociente de inteligencia y puede deberse a los déficit generalizados de las funciones cognitivas, especialmente al déficit específico de la atención. No obstante, al contrario de lo que muestra la literatura (Levin \& Heisenberg, 1979), no se encuentran diferencias significativas entre las pruebas verbales y las manipulativas, siendo la media del $\mathrm{Cl}$ verbal de 87.3 y la del manipulativo de 88.1.

Es sabido que, en general, los pacientes mejoran tras el daño cerebral y sobre todo si participan en programas de neurorehabilitación. La literatura informa de las mejorías que se producen es las funciones motoras, cognitivas y emocionales (Ríos-Lago, Muñoz-Céspedes \& Paul-Lapedriza, 2007). Sin embargo, esto va a depender del patrón de comparación. Efectivamente, como demuestra la literatura, las funciones cognitivas mejoran en general a los dos años si se comparan con los datos de las secuelas de la fase aguda o post-aguda. Pero si comparamos el rendimiento de estas personas con el de la población estándar, el perfil cognitivo cae aproximadamente una desviación típica por debajo de esta última.

Cabe destacar que, aunque un gran porcentaje de los sujetos obtienen puntuaciones no inferiores a una DT de la media en las pruebas que miden inteligencia general, la gran mayoría obtiene resultados patológicos en las que tienen un gran componente de atención compleja, tales como TMT, SDMT o Test de Corsi. Esto implica que, aunque los pacientes estudiados presenten una gran variabilidad en sus secuelas cognitivas, las alteraciones en la función atencional están presentes en todos los sujetos de la muestra, por lo que se puede considerar como el principal déficit de los pacientes con TCE cerrado a los dos años de evolución. No obstante, no se ignora que la principal limitación de este estudio es la ausencia de un grupo control equivalente a la muestra clínica. En vez de esto se compararon los resultados obtenidos con los baremos proporcionados por las diferentes pruebas utilizadas.

Prácticamente la totalidad de los sujetos de la muestra de este estudio había sido incapaz de reiniciar su vida laboral y necesitaban supervisión en las tareas de la vida diaria o eran altamente dependientes. No cabe duda de que a los dos años después del TCE grave los déficit cognitivos merman estas capacidades de adaptación a la vida cotidiana. Esto tiene serias implicaciones en la salud, la familia y la sociedad, y redunda en resaltar la importancia en los programas de rehabilitación neuropsicológica de las funciones cognitivas.

\section{Conclusiones}

El perfil general obtenido es un descenso generalizado en las funciones cognitivas. No se detectaron diferencias atribuibles a la localización de la lesión, predominando las secuelas de carácter difuso. El aspecto fundamental que debemos destacar en nuestro estudio es el deterioro en las funciones atencionales $y$, concretamente, en la atención compleja, que se encuentra significativamente afectada en todos los sujetos evaluados.

\section{Agradecimientos}

Los autores agradecen al Servicio de Neuropsicología y Rehabilitación S. C. (SEREN), Huelva (CIF. J21257373), España. Tel. +34959282643 , la financiación de este trabajo.

\section{Referencias}

Ardila, A., Rosselli, M. y Puente, P. (1994). Neuropsychological evaluation of the Spanish speaker. New York: Plenum Press.

Ariza, M., Pueyo, R. y Sierra, J.M. (2004). Secuelas neuropsicológicas de los traumatismos craneoencefálicos. Anales de Psicología, 20, 303316.

Benton, A. y Hamsher, K. (1977). Multilingual Aphasia Examination Manual. lowa City: University of lowa.

Benton, A.L. (1986). Test de Retención Visual de Benton. Madrid: TEA Ediciones.

Bode, R.K., Heinerman, A.W. y Semik, P. (2000). Measurement propierties of the Galveston Orientation and Amnesia Test (GOAT) and improvement patterns during inpatient rehabilitation. Neuropsychological Rehabilitation, 15, 637-654.

Brown, L., Sherbenou, R.J. y Johnsen, S.K. (1995). Test de Inteligencia no Verbal Toni-2. Madrid: TEA Ediciones.

Caracuel, A. y Cuberos, G. (2009). Los traumatismos craneoencefálicos. En M. Perez, (ed), Manual de neuropsicología clínica (pp. 143-158). Madrid: Pirámide. 
Cullum, C. M. y Bigler, E.D. (1986). Ventricle size, cortical atrophy and the relationship with neuropsychological status in closed head injury: a quantitative analysis. Journal of Clinical and Experimental Neuropsychology, 8, 437-52.

De la Cueva-Barrao, L., Noé-Sebastián, N., Sopena-Novales, P., LópezAznar, D., Ferri-Campos, J., Colomer-Font, C., Martínez-Carsi, C., Abreu-Sánchez, P., Uruburu-García, E., Lull-Noguera, N., RoblesViejo, M. y Chirivella-Garrido, M. (2009). Relevancia clínica de la FDG-PET en los traumatismos craneoencefálicos graves. Revista de Neurología, 49(2), 58-63.

Englander, J., Cifu, D.X., Wright, J.M. y Black, K. (2003). The association of early computed tomography scan findings and ambulation, selfcare, and supervision needs at rehabilitation discharge and at 1 year after traumatic brain injury. Archives of Physical Medicine and Rehabilitation, 84, 214-20.

Ewert, J., Levin, H.S., Watson, M.G. y Kalisky, Z. (1987). Procedural memoryduring posttraumatic amnesia in survivors of severe closed head injury. Implications for rehabilitation. Archives of Neurology, 46, 575-92.

Hanks, R.A., Rapport, L.J., Millis, S. y Deshpande, S.A. (1999). Measures of executive functioning as predictors of functional ability and social integration in a rehabilitation sample. Archives of Physical Medicine and Rehabilitation, 80, 1030-7.

Heaton, R.K., Chelune, G.J, Talley, J.L., Kay, G.G. y Curtiss, G. (2001). Test de clasificación de cartas de Wisconsin. 2 ed. Madrid: TEA Ediciones.

Junqué, C., Bruna, O., Mataró, M. y Puyuelo, M. (1998). Traumatismos craneoencefálicos. Un enfoque desde la neuropsicología y la logopedia. Barcelona: Masson.

Kolb, B. y Whishaw, I.Q. (2006). Neuropsicología Humana. Madrid: Editorial Médica Panamericana.

Ladera, V., Perea, M.V. y Morales, F. (2002). Rendimientos mnésicos en traumatismo craneoencefálico moderado al año. Psicothema, 14(1), 71-76.

Levin, H.S., Grossman, R.G. y Kelly, P.J. (1976). Aphasic disorder in patients with closed head injury. Journal of Neurology Neurosurgery \& Psychiatry, 39(11), 1062-1070.

Levin, H.S. y Heisenberg, H.M. (1979). Neuropsychological outcome of closed head injury. Child's Brain, 5, 281-292.

Lezak, M.D., Howieson, D.B., Loving, D.W., Hannay, H.J. y Fisher, J.F. (2004). Neuropsychological assessment. Oxford: Oxford University Press.

Marshall, S.B., Cayard, C., Foulkes, M.A., Hults, K., Gautille, T. y Charlebois, D.B. (1988). The Traumatic Coma Data Bank: a nursing perspective, Part I. Journal of Neuroscience Nursing, 20, 253-7.

Milner, B. (1986). Interhemispheric dipherences in the localization of psychological processes in man. British Medical Bulletin, 27, 272277.

Millis, S.R., Rosenthal, M., Novack, T.A., Sherer M., Nick, T.G., Kreutzer, J.S., High, W.M. y Ricker, J.H. (2001). Long-Term Neuropsychological Outcome After Traumatic Brain Injury. Journal of Head Trauma Rehabilitation, 16(4), 343-355.

Muñoz-Céspedes, J.M., Paul-Lapedriza, N., Pelegrín-Valero, C. y TirapuUstarruz, J. (2001). Factores de pronóstico en los traumatismos craneoencefálicos. Revista de Neurología, 32(4), 351-364.

Nickels, J.L., Schneider, W. N., Dombovy, M.L. y Wong, T.M. (1994). Clinical use of amantadine in brain injury rehabilitation. Brain Injury, 8, 709-18.

Perea, M.V., Ladera, V. y Morales, F. (1999). Valor predictivo de los tests breves sobre la situación cognitiva en traumatismos craneoencefálicos. Revista de Neurología, 29, 1099-103.

Prigatano, G.P. (1999). Principles of neuropsychological rehabilitation. New York: Oxford University Press.

Reitan, R.M. (1958). Validity of the Trail Making Test as an indication of organic brain damage. Percept Mot Skills, 8, 271-276.

Ríos-Lago, M., Muñoz-Céspedes, J.M. y Paúl-Lapedriza, N. (2007). Alteraciones de la atención tras daño cerebral traumático: evaluación y rehabilitación. Revista de Neurología, 44(5), 291-297.
Sarno, M. T., Buonafura, A. y Levita, E. (1986). Characteristics of verbal impairment in closed head injury patients. Archives of Physical and Medicine Rehabilitation, 67, 400-405.

Shum, D., Sweeper, S. y Murray, R. (1996). Performance on verbal implicit and explicit memory tasks following traumatic brain injury. Journal of Head Trauma Rehabilitation, 11, 43-53.

Smith, A. (2002). Test de Símbolos y Dígitos SDMT. Madrid: TEA Ediciones.

Tabaddor, K., Mattis, S. y Zazula, T. (1984). Cognitive sequelae and recovery course after moderate and severe head injury. Neurosurgery, $14,701-8$.

Tagliaferri, F., Compagnone, C., Korsic, M., Servadei, F. y Kraus, J. (2006). A systematic review of brain injury epidemiology in Europe. Acta Neurochirurgica, 148, 255-268.

Timmerman, M.E. y Brower, W.H. (1999). Slow information processing after very severe closed head injury: impaired access to declarative knowledge and intact application and acquisition of procedural knowledge. Neuropsychologia, 37, 467-78.

Tromp, E. y Mulder, T. (1991). Slowness of information processing after traumatic head injury. Journal of Clinical and experimental Neouropsychology, 13, 821-830.

Valik, E. (2005). The Effect of Moderate to Severe Traumatic Brain Injury (TBI) on Different Aspects of Memory: A Selective Review. Journal of Clinical and Experimental Neuropsychology, 27, 977-1021.

Van Zomeren, A.H. y Van den Burg, W. (1985). Residual complaints of patients two years after severe head injury. Journal of Neurology, Neurosurgery \& Psychiatry, 48, 21-8.

Verger, K., Serra-Grabulosa, J.M., Junqué, C., Álvarez, A., Bartrés-Faz, D. y Mercader, J.M. (2001). Estudio de las secuelas a largo plazo de los traumatismos craneoencefálicos: evaluación de la memoria declarativa y procedimental y de su sustrato neuroanatómico. Revista de Neurología, 33, 30-41.

Von Cramon, D.Y. y Von Cramon, M. (1992). Reflection on the treatment of brain injured patients suffering from problem-solving disorders. Neuropsychogical Rehabilitation, 2, 207-29.

Wardlaw, J.M., Easton, V.J. y Statham, P. (2002). Which CT features help predict outcome after head injury? Journal of Neurology, Neurosurgery \& Psychiatry, 72, 188-92.

Wechsler, D. (1999). Escala de inteligencia para adultos-III. Madrid: TEA Ediciones.

Wilson, B. A. (1992). Rehabilitation and memory disorders. En L.R.S. Squire y N. Butters (Ed), Neuropsychology of memory (pp 315-322). New York: Guilford Press.

Ziino, C. y Ponsford, J. (2006). Vigilance and fatigue following traumatic brain injury. Journal of the International Neouropsychological Society, 12,100 